TRAVAIL

ET EMPLOI

\section{Travail et Emploi}

135 | juillet-septembre 2013

Expérimentations de terrain et politiques publiques du travail et de l'emploi. Apports récents et mises en perspective

\title{
L'expérimentation sociale aléatoire en France en trois questions
}

Three questions about randomized social experimentation in France

\section{Bernard Gomel et Évelyne Serverin}

\section{(2) OpenEdition \\ Journals}

\section{Édition électronique}

URL : https://journals.openedition.org/travailemploi/6081

DOI : 10.4000/travailemploi.6081

ISSN : $1775-416 \mathrm{X}$

Éditeur

DARES - Ministère du Travail

Édition imprimée

Date de publication : 15 septembre 2013

Pagination : 57-71

ISSN : 0224-4365

Référence électronique

Bernard Gomel et Évelyne Serverin, « L'expérimentation sociale aléatoire en France en trois questions », Travail et Emploi [En ligne], 135 | juillet-septembre 2013, mis en ligne le 01 juillet 2015 consulté le 21 septembre 2021. URL : http://journals.openedition.org/travailemploi/6081 ; DOI https://doi.org/10.4000/travailemploi.6081 


\title{
L'expérimentation sociale aléatoire en France en trois questions
}

\author{
Bernard Gomel(*), Évelyne Serverin ${ }^{(* *)}$
}

\begin{abstract}
Dès les années soixante, des outils ont été mis en place en France pour expérimenter les normes avant leur adoption. Cette forme d'évaluation a trouvé un fondement constitutionnel avec la loi du 28 mars 2003 qui autorise l'expérimentation normative, à la fois sur le plan national et sur le plan local. Une telle innovation a été la porte d'entrée d'une forme spécifique d'expérimentation, par assignation aléatoire, qui emprunte ses méthodes aux sciences sociales, et ses terrains aux politiques internationales en matière de pauvreté. Cette forme d'expérimentation soulève trois questions, traitées dans trois parties successives. La première partie relève de la science juridique: cette forme d'expérimentation occupe une place limitée dans l'ensemble des expérimentations normatives. La deuxième est d'ordre scientifique : l'expérimentation sociale n'apporte que peu d'enseignements à la science expérimentale appliquée aux comportements humains. La troisième partie est d'ordre sociopolitique : l'apport de l'expérimentation sociale à l'évaluation des politiques publiques reste à mesurer. La conclusion rappelle les exigences éthiques et scientifiques qui sont posées dans la conduite et l'évaluation des expérimentations portant sur les comportements humains.
\end{abstract}

Dans un système démocratique où les citoyens, via leurs représentants, sont en droit de demander des comptes sur l'usage des deniers publics, l'édiction de règles, et l'évaluation de leur portée, s'entourent d'un appareillage argumentatif où les données chiffrées occupent une place centrale. Ex ante, les chiffres sont mobilisés pour donner des raisons de légiférer, en identifiant les situations dont l'évolution appelle la modification des règles applicables, et en fondant des prédictions sur les effets des règles nouvelles sur la situation visée. Ex post, les évaluations chiffrées visent à mesurer l'effectivité des règles, définie à partir de leurs usages, et à évaluer leur efficacité, mesurée comme l'aptitude de la règle à remplir les objectifs poursuivis. Les évaluations ex ante renvoient aux études préalables, qu'il s'agisse de rationalisation des choix budgétaires (LÉVY-LAMBERT, GuILlaume, 1971), ou à des études d'impact, rendues constitutionnellement obligatoires depuis 2009 pour le vote des projets de lois ${ }^{(1)}$. Les évaluations ex post relèvent du vaste champ de l'évaluation des politiques publiques (VIVERET, 1990), officiellement dévolu au Parlement par l'article 24 de la Constitution(2).

(*) Centre d'études de 1'emploi (CEE); bernard.gomel@ceerecherche.fr

(**) Centre de théorie et analyses du droit (CTAD); eserveri@u-paris10.fr

(1) Article 39 de la Constitution. Loi organique $n^{\circ} 2009-403 \mathrm{du}$ 15 avril 2009 relative à l'application des articles 34-1, 39 et 44 de la Constitution.

(2) «Le Parlement vote la loi. Il contrôle l'action du Gouvernement. Il évalue les politiques publiques.»
C'est surtout sur le terrain de la «préparation» de la norme que les outils se sont développés, et qu'apparaissent, dès les années soixante, des lois et règlements explicitement présentés comme «expérimentaux ». Cette forme d'évaluation a trouvé un fondement constitutionnel avec la loi $n^{\circ}$ 2003-276 du 28 mars 2003 relative à l'organisation décentralisée de la République, qui autorise l'expérimentation normative, à la fois sur le plan national et sur le plan local. Cette innovation a été la porte d'entrée d'une autre forme d'expérimentation, l'expérimentation aléatoire, qui emprunte ses méthodes aux sciences sociales, et ses terrains aux politiques internationales en matière de pauvreté (AlLÈGRE, 2008; Gomel, Serverin, 2009).

Un peu plus de cinq ans après l'entrée en France de l'expérimentation sociale aléatoire dans le périmètre des réformes des politiques sociales, il est légitime de faire le point sur le sens et la portée de cette forme d'évaluation. Pour cela, nous poserons successivement trois questions.

La première question relève de la science juridique : quelle place occupe cette forme d'expérimentation dans le périmètre des expérimentations normatives?

La deuxième question est d'ordre scientifique : quels enseignements apporte-t-elle à la science expérimentale appliquée aux comportements humains? La troisième question est d'ordre sociopolitique : quelle est sa contribution à la conception et à la mise en place des réformes? 
En conclusion, nous reviendrons sur les exigences éthiques et scientifiques qui accompagnent toutes les formes d'expérimentation sur les comportements humains.

\section{Quelle place de l'expérimentation aléatoire dans l'expérimentation normative?}

Si l'on suit la chronologie d'apparition de l'expérimentation dans les politiques publiques en France, on constate que l'Administration a précédé les chercheurs dans la préoccupation d'anticiper les effets des réformes. La constitutionnalisation de cette pratique a ouvert la voie à deux niveaux différents d'expérimentation normative, national et local. L'expérimentation sociale aléatoire est venue s'inscrire sur le plan local, là où se développent les politiques sociales.

\section{L'expérimentation comme anticipation des effets de la norme}

Au départ de la mise à l'épreuve de lois, se trouve l'idée que la décision politique vise des effets positifs d'une réforme mais en redoute les effets négatifs. Ainsi définie, l'expérimentation administrative a une longue histoire derrière elle.

Même si le terme d'expérimentation n'y figure pas, on peut considérer que les lois IVG et RMI ont été les premiers exemples de dispositions mises à l'essai (3) : la loi IVG a suspendu l'application de la loi pénale pendant une durée de cinq ans ; la loi RMI a instauré l'allocation du revenu minimum pour une durée de trois ans ${ }^{(4)}$. À l'issue de la période de validité, le gouvernement présentait un nouveau projet de loi pérennisant les dispositifs.

La pratique de la loi à l'essai a également touché des règles dérogatoires au droit commun concernant des personnes placées dans la même situation, mettant cette fois en jeu le principe d'égalité devant la loi.

Les interventions successives du Conseil constitutionnel sur ces dispositions ont retenu le caractère expérimental des mesures pour justifier les ruptures d'égalité. Dans une décision du 28 juillet 1993, il précisait :

«qu'il est même loisible au législateur de prévoir la possibilité d'expériences comportant des dérogations aux règles ci-dessus définies de nature à lui permettre d'adopter par la suite, au vu des résultats de cellesci, des règles nouvelles appropriées à l'évolution des

(3) Loi n ${ }^{\circ} 75-17$ du 17 janvier 1975, relative à l'interruption volontaire de la grossesse (IVG), loi n ${ }^{\circ} 88-1088$ du $1^{\text {er }}$ décembre 1988 relative au revenu minimum d'insertion (RMI).

(4) Jusqu'en 1979 pour l'IVG (art. 2), jusqu'au 30 juin 1992 pour le RMI (art. 52). missions de la catégorie d'établissements en cause; que toutefois il lui incombe alors de définir précisément la nature et la portée de ces expérimentations, les cas dans lesquels celles-ci peuvent être entreprises, les conditions et les procédures selon lesquelles elles doivent faire l'objet d'une évaluation conduisant à leur maintien, à leur modification, à leur généralisation ou à leur abandon. »

Une deuxième décision du 6 novembre 1996, relative à l'information et à la consultation des salariés dans certaines entreprises, réitérait ces exigences à propos de la possibilité pour les partenaires sociaux de déroger au droit commun de la négociation collective, en raison de son caractère expérimental, et qui prévoyait que le Gouvernement présente dans les cinq ans un rapport au Parlement ${ }^{(5)}$.

À ces pratiques législatives se sont ajoutées celles du pouvoir exécutif, consistant à édicter des normes limitées à la fois dans l'espace et dans le temps.

Ainsi, l'atteinte au principe d'égalité n'a pas été retenue dans le cas de l'application progressive d'un contrôle sanitaire aux entreprises alimentaires, faute de vétérinaires en nombre suffisant ${ }^{(6)}$. De même, un décret de 1968 instituant un juge des mises en état devant certains tribunaux ou cours d'appel n'a pas été considéré comme violant le principe d'égalité devant la loi dans la mesure où cette limitation avait un caractère provisoire ${ }^{(7)}$. Enfin, dans un avis du 24 juin 1993, le Conseil d'État estimait que des dispositions tarifaires différentes entre usagers de lignes de la SNCF répondant aux mêmes caractéristiques ne sauraient se perpétuer au-delà d'une période expérimentale d'un an, période qui devait être mise à profit pour définir des critères objectifs du nouveau régime tarifaire $^{(8)}$.

La validité des dispositions légales ou réglementaires dépendait donc de l'existence d'une évaluation, conduite par l'autorité qui édicte la norme, et matérialisée par un rapport.

\section{La consécration constitutionnelle de l'expérimentation normative}

Après quarante ans de mise à l'essai de textes de toutes natures, il n'était plus possible de poursuivre dans cette voie sans donner aux pratiques un support constitutionnel. Dans une décision du 17 janvier 2002, le Conseil constitutionnel considérait «qu'en ouvrant au législateur, fût-ce à titre expérimental, dérogatoire et limité dans le temps, la possibilité d'autoriser la collectivité territoriale de Corse à prendre des mesures relevant du domaine de la loi,

(5) Cons. const., déc. 6 nov. 1996, n 96-383 DC.

(6) CE, Sect., 13 oct. 1967, n 64778, Rec. CE., p. 365 ; Revue de droit public, 1968, p. 408.

(7) CE, 21 fév. $1968, n^{\circ} 68615$ et s., Ordre des avocats près la cour d'appel de Paris, Rec. CE., p. 123; Dalloz, 1968, p. 222.

(8) CE, Ass. gén., sect. TP, 24 juin 1993, avis nº 353605. 
la loi déférée est intervenue dans un domaine qui ne relève que de la Constitution ${ }^{(9)}$ \.

À la suite de cette décision, la loi constitutionnelle $\mathrm{n}^{\circ}$ 2003-276 du 28 mars 2003 relative à l'organisation décentralisée de la République (que certains ont dénommée «L'acte II » de la décentralisation »; JANIN, 2005), autorisait l'expérimentation normative à la fois sur le plan national et sur le plan local. D'une part, elle insérait dans la Constitution un article 37-1: «La loi et le règlement peuvent comporter, pour un objet et une durée limités, des dispositions à caractère expérimental»; d'autre part, elle ajoutait à l'article 72 de la Constitution relatif aux collectivités territoriales un alinéa quatre leur ouvrant la possibilité de « déroger, à titre expérimental et pour un objet et une durée limités, aux dispositions législatives ou réglementaires qui régissent l'exercice de leurs compétences». En application de cette dernière disposition, la loi organique $n^{\circ}$ 2003-704 du 1er août 2003 a ajouté au Code général des collectivités territoriales un chapitre spécifique dédié aux expérimentations, dont elle détermine le cadre général ${ }^{(10)}$.

Les observateurs notent que l'inscription de l'expérimentation dans la Constitution n'a suscité ni controverses ni débats très nourris (STAHL, 2010). Il faut voir dans ce relatif silence la preuve de l'existence d'une forme de consensus, forgé au cours de quarante ans de pratiques, sur l'intérêt de tester les normes avant de les généraliser. Mais en consacrant les vertus de la «prudence normative», les textes constitutionnels ont ouvert la porte à la méthode expérimentale, dont il n'avait jamais été question jusqu'alors.

\section{Des usages de l'autorisation constitutionnelle}

Depuis l'entrée en vigueur de la révision constitutionnelle de 2003, c'est l'article 37-1 qui a été le plus sollicité, dans une logique principalement administrative. L'expérimentation sociale s'est inscrite, quant à elle, sur le fondement de l'article 72 .

(9) Cons. const., 17 janv. 2002, $\mathrm{n}^{\circ}$ 2001-454, statut de la Corse, note J.-E. Schoettl : L'actualité juridique : droit administratif, 2002, p. 100 et $\mathrm{s}$.

(10) «La loi qui autorise, sur le fondement du quatrième alinéa de l'article 72 de la Constitution, les collectivités territoriales à déroger, à titre expérimental aux dispositions législatives régissant l'exercice de leurs compétences, définit l'objet de l'expérimentation ainsi que sa durée, qui ne peut excéder cinq ans, et mentionne les dispositions auxquelles il peut être dérogé. La loi précise également la nature juridique et les caractéristiques des collectivités territoriales autorisées à participer à l'expérimentation ainsi que, le cas échéant, les cas dans lesquels l'expérimentation peut être entreprise. Elle fixe le délai dans lequel les collectivités territoriales qui remplissent les conditions qu'elle a fixées peuvent demander à participer à l'expérimentation» (article L. O. 1113-1).

\section{Les expérimentations administratives sur le fondement de l'article 37-1}

Dans la tradition des textes à l'essai, l'expérimentation fondée sur l'article 37-1 est tournée vers l'action administrative elle-même, dans ses dimensions techniques et budgétaires.

Le premier usage de l'autorisation constitutionnelle a été la loi du 13 août 2004 relative «aux libertés et aux responsabilités locales» (JANIN, 2005). Ce texte a élargi le domaine des expérimentations à l'occasion des transferts et délégations de responsabilités et de compétences, qu'il s'agisse de gestion de crédits ou d'exercice de compétences. Saisi d'un recours contre le texte, le Conseil constitutionnel réitère sur ce nouveau fondement les critères forgés au fil des décisions antérieures ${ }^{(11)}$.

Dans une autre décision du 13 décembre 2007, le Conseil constitutionnel précise que «l'article 37-1 de la Constitution permet que la loi de financement de la sécurité sociale inclue des dispositions expérimentales [...], mais que le Conseil serait amené à censurer des dispositions qui $[\ldots]$ seraient en réalité non pas d'application expérimentale, mais d'application bornée dans le temps ${ }^{(12)} \gg$. Ce considérant exprime une idée essentielle : on peut expérimenter des dispositions, à condition qu'elles soient accompagnées d'une évaluation qui prenne la mesure de toutes les conséquences de la réforme.

D'autres réformes, poursuivant des finalités économiques ou sociales ou environnementales, sont coûteuses, et nécessitent un passage par un «sas» expérimental. Les exemples sont légion ${ }^{(13)}$.

(11) Cons. const., 12 août 2004, $\mathrm{n}^{\circ}$ 2004-503 DC : «L'article 37-1 de la Constitution, issu de la révision constitutionnelle du 28 mars 2003 susvisée, permet au Parlement d'autoriser, dans la perspective de leur éventuelle généralisation, des expérimentations dérogeant, pour un objet et une durée limités, au principe d'égalité devant la loi. [...] Toutefois, le législateur doit en définir de façon suffisamment précise l'objet et les conditions et ne pas méconnaître les autres exigences de valeur constitutionnelle.»

(12) Cons. const., 13 décembre 2007, n 2007-558 DC - Loi de financement de la sécurité sociale.

(13) Les articles 37-1 et 38 de la Constitution ont été visés par l'ordonnance $\mathrm{n}^{\circ}$ 2006-433 du 13 avril 2006, «expérimentant le contrat de transition professionnelle», pour des bassins d'emploi déterminés (dispositif abrogé par la loi n 2011-893 du 28 juillet 2011 pour le développement de l'alternance et la sécurisation des parcours professionnels). Sur le même visa, la loi $\mathrm{n}^{\circ} 2008-596$ du 25 juin 2008, portant modernisation du marché du travail, a instauré à titre expérimental pour une durée de cinq ans un nouveau contrat de travail, dit à objet défini (art. 6). La loi n 2009-1673 du 30 décembre 2009 de finances pour 2010 a introduit à titre expérimental un revenu contractualisé d'autonomie. Le décret $n^{\circ} 2010-1395$ du 12 novembre 2010 relatif à la médiation et à l'activité judiciaire en matière familiale a mis en place une expérimentation d'injonction de rencontrer un médiateur familial. La loi $\mathrm{n}^{\circ}$ 2011-1862 du 13 décembre 2011 relative à la répartition des contentieux et à l'allégement de certaines procédures juridictionnelles a prévu l'expérimentation d'une tentative de médiation familiale obligatoire dans certains litiges concernant les enfants. 
Dernière-née de cette série, la mise en place de citoyens assesseurs dans les affaires correctionnelles par la loi $\mathrm{n}^{\circ}$ 2011-939 du 10 août 2011, qui a donné lieu à un rapport (négatif) établi par deux magistrats de la Cour de cassation (SALVAT, BOCCONGibod, 2013).

Dans tous ces domaines, l'expérimentation porte sur la norme elle-même, et son insertion dans l'ensemble des normes, sur ses incidences sociales, économiques, et budgétaires. La charge de l'expérimentation repose sur l'Administration elle-même, sans appel à des recherches extérieures, et surtout, sans qu'aucune hypothèse scientifique ne soit testée. Ainsi, il n'est pas prévu dans le dispositif expérimental des citoyens assesseurs de tester la «sévérité» des non-magistrats, alors même que cette idée avait constitué un des motifs de la réforme. Ce sont seulement les conséquences de cette introduction qui doivent être évaluées. Bref, le choix de l'expérimentation revient à introduire une sorte de «clause de repentir» pour ne pas engager l'avenir sur des dispositifs coûteux, controversés, ou qui présentent des risques sérieux de détournement.

\section{Les expérimentations aléatoires sur le fondement de l'article 72}

Ce n'est pas dans le terreau de l'article 37-1 que l'expérimentation randomisée va plonger ses racines, mais dans celui de l'article 72 de la Constitution. Pour la première fois, l'objet de l'expérimentation n'était pas de vérifier la qualité de la norme, ni la faisabilité de la réforme, ni même son coût, mais de mesurer ses effets sur le comportement des assujettis.

Si l'expérimentation sociale avec assignation aléatoire a une longue histoire internationale, elle commence en France avec le rapport de la commission Familles, vulnérabilité, pauvreté, (2005), présidée par Martin Hirsch, proposant au gouvernement «des expérimentations selon des programmes qu'il définirait lui-même mais qu'il souhaiterait tester sur une partie du territoire» (Gomel, Serverin, 2009, 2011). Le législateur va lui donner satisfaction en deux temps. D'abord avec l'article 142 de la loi de finances pour $2007 \mathrm{du}$ 21 décembre 2006 :

«À titre expérimental, afin d'améliorer les conditions d'incitation financière au retour à l'emploi et de simplifier l'accès aux contrats de travail aidés, les départements mentionnés par le décret prévu à l'article L. O. 1113-2 du Code général des collectivités territoriales sont autorisés, pour une durée de trois ans à compter de la date de publication dudit décret, à adopter, en faveur des bénéficiaires du revenu minimum d'insertion, tout ou partie des dérogations aux dispositions du Code du travail et du Code de l'action sociale et des familles $[\ldots]$ ».
Le 4 mai 2007, deux départements, celui de l'Eure et celui de la Côte-d'Or, qui s'étaient respectivement portés volontaires le $1^{\mathrm{er}}$ février et le 23 mars 2007, ont été autorisés par décret à procéder à des expérimentations portant, pour le premier, sur l'amélioration des conditions d'incitation financière au retour à l'emploi; pour le second, sur la simplification de l'accès aux contrats aidés. Le 20 juin 2007, le conseil général de l'Eure adoptait une délibération fixant les règles dérogatoires applicables à l'expérimentation dans le département. Sans attendre le terme de cette première expérimentation, le législateur en a modifié substantiellement l'économie en passant, à l'été 2007, à une seconde étape. Les articles 18 à 23 de la loi du 21 août 2007 dite «Tepa» (loi en faveur du travail, de l'emploi et du pouvoir d'achat) ont ouvert l'expérimentation du revenu de solidarité active (RSA) aux départements volontaires. L'article 22 de la loi précitée imposait au gouvernement le dépôt d'un rapport d'évaluation avant toute généralisation $\mathrm{du}$ RSA. Le rapporteur du projet de loi généralisant le RSA se réjouissait ainsi de la démarche suivie pour conduire cette évaluation : «Un comité d'évaluation a été mis en place, sous la présidence de M. François Bourguignon, et a établi dès les débuts de l'expérimentation, et non a posteriori comme trop souvent, un protocole d'évaluation reposant sur la comparaison de zones témoins avec les zones d'expérimentation $\left.{ }^{(14)}{ }\right)$.

Le modèle de l'expérimentation randomisée s'est donc inscrit dans le cadre de l'autorisation constitutionnelle de dérogation locale à la règle, comme simple méthode d'évaluation. Son emprise s'est élargie sous couvert de la démarche de «l'expérimentation sociale», présentée en France comme une innovation majeure capable de trancher des questions sociales complexes indéfiniment débattues sans conclusion (COMmission Famille, VULNÉRABILITÉ, PAUVRETÉ, 2005). Mais elle est loin d'avoir remplacé les expérimentations administratives traditionnelles qui, seules, permettent d'anticiper les effets d'une réforme dans toutes ses dimensions, sans céder aux sirènes du changement. C'est sur ce point que les deux formes d'évaluation divergent. L'expérimentation administrative est portée par une défiance normative: on veut anticiper les conséquences négatives d'une réforme décidée. L'expérimentation aléatoire est guidée par une espérance normative : on attend des effets positifs d'une réforme projetée.

(14) Daubresse M.-P. (rapporteur) (2008), «Rapport fait au nom de la commission des affaires culturelles, familiales et sociales sur le projet de loi $\left(n^{\circ} 1100\right)$ généralisant le revenu de solidarité active et réformant les politiques d'insertion", Rapport, $\mathrm{n}^{\circ} 1113$, Paris, Assemblée nationale, p. 35 ; disponible en ligne à l'adresse: http://www.assemblee-nationale.fr/13/ pdf/rapports/r1113.pdf; consulté le 2 septembre 2013. 


\section{Quels enseignements pour les sciences sociales expérimentales?}

La science économique est familière de l'idée d'expérimentation. Dans l'économie standard, l'expérimentation forme un des « volets empiriques » des modèles microéconomiques, dont elle vise à reproduire le fonctionnement en laboratoire. Ce champ a pris une importance considérable depuis ses débuts dans l'immédiat après-guerre, au point de justifier 1'attribution en 2002 du prix Nobel d'économie à Vernon L. Smith «pour avoir fait de l'expérience en laboratoire un instrument d'analyse économique empirique» (BIAIS, Rullière, 2003). Cependant, si cette forme d'expérimentation comporte une dimension d'aide à la décision, notamment en matière de politique économique, son ambition première est la connaissance, soit pour tester la validité des théories économiques, soit pour expliciter des régularités observées.

L'expérimentation prend une autre tournure lorsque, sans se borner à fournir des modèles théoriques "validés» au politique, elle vise à tester l'efficacité d'un instrument d'intervention donné pour l'obtention d'un résultat déterminé. La démarche relève alors de la science expérimentale, et exige le recours à une méthode robuste de constitution des groupes. L'assignation aléatoire, théorisée par Ronald Fischer dès 1935, est la meilleure candidate à la conduite de ce type de recherche. Elle a le mérite de rendre identiques, avec une précision qui ne dépend que de leur taille, les différents échantillons ainsi constitués comme le rappellent systématiquement les «randomisateurs» :

«Le tirage au sort, au sein d'une même population, d'un groupe test et d'un groupe témoin permet d'assurer la comparabilité des deux groupes: en moyenne, les caractéristiques de la population de chacun de ces groupes ne sont pas statistiquement différentes; plus l'échantillon est grand, plus ces caractéristiques ont de chance d'être proches. Cette propriété vaut pour les caractéristiques observables (sexe, âge, résultats scolaires, niveau de diplôme, etc.) mais également celles qui ne le sont pas : caractéristiques particulières des individus et difficiles à mesurer, telles que la motivation, la confiance en soi, etc. ${ }^{(15)}$ 》

Le strict respect de l'assignation aléatoire protège ainsi des biais de sélection qui viennent perturber la valeur et la précision des estimations réalisées à partir des données d'une expérience. Les domaines

(15) Ministère des Sports, de la Jeunesse, de l'Éducation populaire et de la Vie associative, Fonds d'expérimentation pour la jeunesse (2012), Rapport d'activité 2009-2011, p. 35. Disponible en ligne à l'adresse : http://www.experimentation. jeunes.gouv.fr/IMG/pdf/FEJ_RA_20092011_CorpsRapport. pdf; consulté le 2 septembre $20 \overline{13}$. La taille des échantillons dépend d'autres facteurs comme l'importance de l'effet, cf. infra. étudiés par FISCHER relevaient de la science expérimentale : l'affectation aléatoire y est affaire de procédure, de méthode, tout comme la qualité du recueil des données de l'expérimentation. Lorsque l'expérimentation a été conduite selon les règles de l'art, l'écart entre le critère mesuré sur les groupes traités et les non-traités est l'effet propre du traitement sur le critère.

Il est tentant de considérer les différentes politiques sociales comme des «traitements» appliqués à des populations dont on veut changer les comportements. Face aux autres modes de preuve de l'efficacité d'une réforme, les «randomisateurs» apportent la promesse d'une plus grande certitude scientifique quant aux effets d'un dispositif sur des comportements donnés. Cependant, dès lors que les expérimentations se déroulent dans le monde réel et non en laboratoire, la preuve est plus difficile à établir. Comme nous allons le voir, plusieurs exemples d'expérimentations conduites en France au cours de la période récente montrent que leurs promoteurs n'ont pas porté toute l'attention requise aux conséquences de leur immersion dans le monde réel sur la robustesse des résultats. Dans le cas du RSA, il apparaît d'abord que l'évaluateur n'a été qu'un observateur de l'expérience, sans avoir eu à aucun moment la maîtrise de la conduite des opérations. Dans l'expérimentation sur le placement de chômeurs, il n'a pas été en mesure de contrôler jusqu'au bout l'opération d'assignation aléatoire. Enfin, dans le cas de l'expérimentation de la «Mallette des parents», il n'a pas pu isoler statistiquement l'effet propre du dispositif.

\section{La difficile maîtrise de la conduite de l'expérimentation}

L'apport de l'expérimentation des trente-trois RSA départementaux à la preuve de l'effet de l'incitation financière sur le retour à l'emploi a été controversé dès la discussion parlementaire à l'automne 2008 (Gomel, Serverin, 2009), et discuté par certains économistes (CAHUC, ZYLBERBERG, 2009). Malgré la très courte durée de l'expérimentation (cinq mois), les expérimentateurs ont apporté au débat un «résultat» d'une grande portée argumentative : l'augmentation du taux de retour à l'emploi est de $30 \%$. Quelques mois plus tard, lorsque les résultats ont été connus sur une période un peu plus longue, l'effet du RSA est trois fois plus faible. Quel que soit son niveau, ce pourcentage n'a pas le sens que les parlementaires ont pu lui donner, mais un sens purement statistique, résumé en ces termes par le Comité scientifique d'évaluation: "Le taux d'entrée en emploi des allocataires du RMI dans les zones expérimentales est en moyenne plus élevé que dans les zones témoins, mais l'écart varie assez fortement entre départements et selon les périodes » (COMITÉ D'ÉVALUATION DES EXPÉRIMENTATIONS, 2009, p. 12). 
Néanmoins, l'écart entre zones expérimentales et zones témoins, qui était de $30 \%$ en moyenne sur les cinq premiers mois, n'est plus que de $9 \%$ sur quinze mois, à la limite de la significativité au seuil usuel de $5 \%$ (ibid., p. 13) :

«[...] le taux mensuel moyen d'entrée en emploi en zones témoins est de 3,1\%. L'écart entre zones expérimentales et zones témoins est de 0,28 point de pourcentage, soit un surcroît de $9 \%$ du taux d'entrée en emploi dans les zones expérimentant le rSa. [...] la probabilité de se tromper en affirmant que l'effet du rSa expérimental sur le retour à l'emploi est supérieur à zéro est de $12 \%$, une valeur un peu élevée mais qui laisse néanmoins une place limitée à l'incertitude.»

Ce n'est pas la seule faiblesse de l'expérimentation. Dès l'entrée en vigueur du dispositif, un phénomène massif de non-recours à sa partie nouvelle, le RSA-activité, visant les travailleurs pauvres, fait douter de la pertinence d'un modèle orienté vers la seule incitation financière (SERVERIN, 2012).

Au-delà de la discussion des résultats, deux points de méthode soulèvent des interrogations.

D'une part, la méthode de constitution des zones tests et témoins a échappé aux évaluateurs, ce qui est particulièrement gênant sachant le prix que les expérimentateurs attachent à l'assignation aléatoire. La loi a en effet laissé aux collectivités territoriales la liberté de choisir les modalités concrètes de l'expérimentation. En particulier, les conseils généraux ont choisi le territoire de l'expérimentation, souvent le plus en difficulté socialement, sans se soucier de la possibilité de trouver dans le département un territoire témoin à lui comparer. L'équipe chargée de sélectionner les zones témoins n'a pas pu associer à chaque zone testée une zone témoin. Il ne leur a donc pas été possible de comparer le taux de retour à l'emploi de chaque RSA départemental expérimenté à celui du RMI, ce qui aurait permis de tirer des enseignements des variations entre RSA expérimentaux. Comme les populations traitées et témoins n'ont été comparables que globalement, les seuls résultats calculables en matière de retour à l'emploi ont porté sur l'écart moyen entre l'ensemble des traités et l'ensemble des témoins.

En second lieu, les études qualitatives de suivi de l'expérimentation ont montré que l'information des personnes concernées dans les territoires par le RSA expérimental n'a pas été davantage contrôlée. Or c'est pourtant là que se situe le levier principal de l'incitation recherchée par les promoteurs de l'expérimentation. Une étude monographique menée pendant l'expérimentation dans cinq des départements (LONCLE et al., 2008) a montré que la communication auprès des publics visés a été «prudente», pour ne pas dire opaque, alors que les intéressés auraient dû disposer d'une information complète. Les objectifs eux-mêmes ne se limitaient pas à la reprise d'activité, mais pouvaient être plus larges (modifier l'action des accompagnateurs, assurer la prévisibilité des revenus, etc.), dans la continuité des réorganisations déjà engagées dans le processus de décentralisation du RMI (16).

En réalité, les conditions de l'expérimentation n'ont été contrôlées qu'à la marge, ce qui aurait dû inciter les évaluateurs à la prudence, et à tout le moins, les conduire à refuser de fournir quelque résultat que ce soit sur le caractère «incitatif» du dispositif.

\section{Le difficile contrôle des objectifs de l'assignation aléatoire}

Une importante expérimentation aléatoire menée en 2007 par l'Unedic et l'ANPE (Agence nationale pour l'emploi) sur l'accompagnement renforcé des demandeurs d'emploi montre le changement de la place de l'aléa dans les expérimentations, les raisons de ce déplacement et ses conséquences en termes de résultats.

L'évaluation de l'efficacité de deux procédures d'accompagnement renforcé des demandeurs d'emploi était une première en France par les moyens exceptionnels qu'elle a mobilisés (l'évaluation porte en tout sur plus de 200000 demandeurs d'emploi) et par sa qualité méthodologique. Sur la base d'un tirage au sort, trois groupes ont été constitués : «ceux auxquels on propose l'accompagnement OPP [opérateur privé de placement], ceux auxquels on propose l'accompagnement CVE [Cap vers l'entreprise], et ceux auxquels on propose l'accompagnement renforcé classique» (ANPE, DARES, UNEDIC, 2008, p. 4). Les auteurs soulignent que le tirage au sort garantit la comparabilité de ces trois groupes (p. 4) :

«Même proportion de jeunes, de femmes, etc. (on peut le vérifier), mais aussi même proportion de demandeurs d'emploi motivés, dynamiques, etc. (même si on ne peut pas le vérifier, faute de mesurer vraiment la motivation ou le dynamisme, on est sûr que c'est le cas à partir du moment où les groupes sont suffisamment grands et sont tirés au hasard). Par conséquent, si on observe des différences de retour en emploi entre ces trois groupes, on sait que ces différences sont dues au fait qu'on ne leur a pas proposé le même accompagnement, et à cela seulement. »

Malgré cette présentation, tout le monde retiendra de cette expérimentation la supériorité de

(16) Une autre enquête, réalisée cette fois par le Credoc (Centre de recherche pour l'étude et l'observation des conditions de vie; Alberola et al., 2008) auprès des bénéficiaires dans les cinq mêmes départements, montre que les allocataires sont bien loin de se situer dans l'alternative entre travail et assistance que leur prêtent les modèles. La préoccupation qui se fait jour est celle de la régularité et de la certitude du versement des allocations, comme complément de salaire. Bref, s'exprime un besoin de sécurité et de prévisibilité que partagent tous les ménages, quel que soit leur niveau de revenu. 
l'opérateur public (CVE) sur les opérateurs privés, sur fond de compétition entre l'ANPE et l'Unedic pour se voir confier le marché des services du placement des chômeurs (17). Comment expliquer ce malentendu, alors que la comparaison directe entre CVE et OPP n'était tout simplement pas au programme de l'expérimentation (18)? Il semble qu'il y ait eu un flottement dans les objectifs de l'expérimentation au sein même du rapport final (BeHAGHel et al., 2009).

D'un côté, le rapport rappelle l'objectif initial (p. IV) : «Ce protocole, dit "d'expérimentation contrôlée", garantit que les différences de situation sur le marché du travail que l'on peut observer entre les groupes après plusieurs mois ne peuvent résulter que du bénéfice des dispositifs. On mesure ainsi avec précision et certitude la plus-value des programmes OPP et CVE.» Le taux de sortie vers l'emploi à trois, six et douze mois du flux indemnisable en l'absence d'accompagnement renforcé est donné : $12 \%$ à trois mois, $23 \%$ à six mois et $37 \%$ à douze mois. "Au bout de 12 mois, CVE permet de passer le taux de sortie de $37 \%$ à $44 \%$ » et «les effets des OPP [...] sont globalement plus faibles et plus tardifs» $\gg(\mathrm{p} . \mathrm{V})$.

D'un autre côté, on insiste sur la comparaison directe entre les deux opérateurs. Pour cela, on restreint l'observation aux territoires communs qui assurent que «les conditions économiques et les populations cibles sont alors identiques» (p. V). «Dans ces zones, les effets des deux programmes sont plus forts, mais aussi plus contrastés : CVE permet d'augmenter de 11 points les taux de sortie dès le $3^{\mathrm{e}}$ mois, tandis qu'à cet horizon l'effet des OPP est toujours non significatif. À 12 mois, CVE a un impact de 8,5 points et les OPP un impact de 6,4 points 》 (p. V).

D'une partie à l'autre du rapport, l'objectif de l'évaluation a changé, du comportement des demandeurs d'emploi à celui des opérateurs eux-mêmes. Ce glissement s'explique par les conditions dans lesquelles l'expérimentation a été menée et, plus précisément, par l'intervention directe des opérateurs dans l'assignation finale des demandeurs d'emploi aux groupes testés. En effet, selon l'étude, seulement $47 \%$ des demandeurs d'emploi aléatoirement affectés à un OPP ont été effectivement pris en charge par cet OPP, le taux de prise en charge étant de $43 \%$ en CVE. Et seule une

(17) Avant que la fusion ANPE-Assedic ne vienne bouleverser la répartition des rôles qui s'ébauchait alors.

(18) Il est seulement possible de comparer d'une part OPP et traitement standard et de l'autre, CVE et traitement standard. En revanche, il n'est pas possible de traiter directement l'écart entre résultats OPP et résultats CVE en conservant la force et la sécurité apportées par l'aléa. Cette comparaison ne peut être qu'indirecte; il y a eu en fait deux expérimentations parallèles pour comparer les performances respectives de CVE et d'OPP par rapport au traitement standard. petite proportion des non-entrées, moins de $20 \%$, s'explique par un refus des demandeurs d'emploi. Dans la grande majorité des cas, les demandeurs d'emploi ont été écartés à la suite d'une sélection organisée directement, au cas par cas, par les opérateurs, qui ont tenté de ne conserver que les demandeurs d'emploi les plus susceptibles, à leur sens, de profiter de l'accompagnement renforcé. La plus grande sélectivité de CVE pourrait s'expliquer par une meilleure connaissance des demandeurs d'emploi et expliquer la plus grande réussite de leurs accompagnements renforcés. Ce point est abordé en toute fin de la synthèse du rapport final d'évaluation, comme un élément nouveau ajouté à la réflexion sur les perspectives futures alors qu'il relance en réalité les interrogations sur la capacité de l'affectation aléatoire, qui est l'innovation principale de la méthode, à rendre traduisibles directement en effets propres des programmes les écarts constatés entre les taux de sortie vers l'emploi. Comme le disent pour terminer les auteurs, «il est plausible que les différences de résultats [des OPP] par rapport au programme CVE puissent s'interpréter par les incitations données aux différents acteurs » (p. VIII).

Malgré les aménagements très importants que l'assignation aléatoire a connus dans la mise en œuvre de l'expérimentation, le rapport final continue de mettre l'accent, dans sa présentation du protocole expérimental, sur son importance (pp. 2-3) :

«La plus-value d'un programme est définie comme la différence entre la situation des individus bénéficiant du dispositif et ce qui aurait été leur situation s'ils n'en avaient pas bénéficié [...]. Pour reconstituer cette situation hypothétique (ou contre-factuelle), on fait en général référence à un groupe témoin. [...] Construire un groupe témoin approprié est difficile. [...] Il est néanmoins possible de s'assurer, par construction, qu'on dispose d'un groupe de bénéficiaires et d'un groupe témoin rigoureusement comparables. Pour cela, il suffit de les tirer au sort au sein d'une même population. Les différences de trajectoires qu'on observe une fois le programme mis en œuvre peuvent alors être attribuées de façon transparente et robuste au programme et à lui seul. Tel est le principe du protocole expérimental appliqué à l'évaluation des programmes OPP et CVE...»

La présentation finale, une fois l'expérimentation conduite, reprend très exactement les termes utilisés pour annoncer l'évaluation (ANPE, DARES, UNÉDIC, 2008), malgré les «aléas» rencontrés lors de la mise en œuvre.

Les évaluateurs ont négligé l'impact de la sélection des opérateurs sur la portée de l'accompagnement renforcé, pour ne se préoccuper que de l'adhésion volontaire des demandeurs d'emploi (compliance) : «L'évaluation renseigne uniquement sur l'effet moyen de l'accompagnement renforcé lorsque l'adhésion est volontaire et que seulement $50 \%$ de ceux auxquels le programme est proposé y 
adhèrent; l'effet moyen sur l'ensemble des demandeurs d'emploi si l'adhésion était obligatoire et si $100 \%$ des demandeurs adhéraient serait potentiellement différent»(p. 14). Ce qui est finalement calculé, c'est un effet moyen du traitement sur les seuls traités (PARIENTÉ, 2008). Les conditions concrètes de l'entrée effective ${ }^{(19)}$ dans les dispositifs d'accompagnement sont considérées comme une donnée de l'expérimentation. C'est pourtant le sens même "d'effet du traitement», qui se trouve ainsi modifié.

\section{Isoler l'effet propre de la variable étudiée}

Le dispositif «Mallette des parents» mis en œuvre en 2008-2009 dans le cadre du Fonds d'expérimentation pour la jeunesse (FEJ) et évalué par une équipe de l'École d'économie de Paris, est porteur de la même promesse d'amélioration d'une situation sociale problématique: l'absentéisme scolaire (Avvisati et al., 2011). Le financement de cette expérimentation est justifié par le fait que l'implication des parents d'élèves au collège et ses effets sur le comportement des élèves est une question cruciale, longtemps débattue et que, «pourtant, rien n'a jamais été vraiment tenté pour l'éclairer rigoureusement» (p. 5). Pour la première fois en France, une intervention expérimentale portant sur cette question permettrait «de juger de l'efficacité d'une politique» et « de la pertinence de la généraliser» (p. 5), grâce à un protocole expérimental réalisant «une évaluation rigoureuse et transparente» (p. 5) du programme. Selon les expérimentateurs, "comme le tirage au sort assure qu'il n'existe aucune différence systématique, $[\ldots]$ les différences que l'on peut observer $[\ldots]$ peuvent être sans aucun doute attribuées à une cause unique : le bénéfice du programme» (p. 5).

L'intervention s'est déroulée dans une quarantaine de collèges de l'académie de Créteil (pp. 3-4).

«En début d'année, une centaine de classes environ ont été tirées au sort dont les parents ont été ensuite invités par le principal à participer à une série de réunions d'information sur le fonctionnement du collège et d'échanges sur la meilleure façon d'aider les enfants et d'interagir avec les enseignants. En fin d'année scolaire, lorsqu'on compare ces parents à ceux des autres classes de sixième (initialement similaires, mais non tirées au sort), ils se caractérisent par un surcroît d'implication très net dans

(19) L'acceptation des demandeurs d'emploi assignés au hasard à un accompagnement renforcé de CVE ou d'un OPP pose seulement un problème de généralisation des résultats de l'expérimentation. En revanche, la sélection supplémentaire par les opérateurs pose un autre problème : les effets observés vont mélanger deux causes : la qualité de leur accompagnement renforcé d'une part et, d'autre part, la capacité des opérateurs à ne retenir, parmi les demandeurs d'emploi assignés aléatoirement et volontaires, que les plus aptes à mettre à profit le renforcement des prestations pour accéder à l'emploi. Et pour réaliser cette seconde sélection, CVE disposait certainement d'une meilleure expertise des données ANPE mises à la disposition de tous les opérateurs. les scolarités. [...] Surtout, les données recueillies révèlent que ce surcroît d'implication se traduit par une amélioration très sensible du comportement des enfants. »

Cette amélioration a été mesurée à l'aide d'une série d'indicateurs du comportement des élèves portant sur l'absentéisme, les sanctions, les «distinctions» et les notes de vie scolaire. "Qu'il s'agisse de l'absentéisme ou du score synthétique "qualité du comportement" résumant les trois autres variables, l'avantage du groupe test sur le groupe témoin est de l'ordre de $10 \%$ d'un écart-type. Par leur amplitude, ces différences se distinguent sans ambiguiité d'un pur aléa d'enquête» (p. 15). De plus, l'expérimentation aurait établi «que l'effet vertueux de ces politiques d'ouverture en direction des parents s'étend au-delà du cercle habituel des familles les plus attentives aux scolarités de leurs enfants pour atteindre (par un effet d'entraînement dans les classes) les familles et les élèves les plus éloignés du système scolaire» (p. 3).

Mais comment explique-t-on cette amélioration? Pour les expérimentateurs, il ne fait pas de doute que c'est un effet du programme d'implication des parents. Cependant, il est loin d'être sûr que la population traitée ait été davantage impliquée dans la vie du collège. On relève dans les résultats un taux équivalent de non-réponses des parents des groupes tests et des groupes témoins à l'évaluation finale, alors qu'on aurait attendu une meilleure participation des premiers. C'est le point fragile de la méthodologie que reconnaissent les expérimentateurs mais dont ils limitent la portée : «Si les taux de réponses étaient très différents [...], la comparaison des réponses recueillies dans les classes tests et les classes témoins cesserait de pouvoir s'interpréter comme reflétant le seul effet de l'éligibilité au programme expérimental» (p. 10). Or, même non significatif, le fait que le taux de réponses des parents des classes tests soit toujours légèrement inférieur à celui des classes témoins est gênant pour une expérimentation qui porte précisément sur l'implication des parents et son effet sur le comportement des enfants.

On le voit, il n'est pas si aisé dans un cadre expérimental «naturel» d'assurer la validité des résultats, d'un point de vue interne (au regard des variables retenues) et, plus encore, d'un point de vue externe, en raison de l'impossibilité d'être certain que d'autres variables causales ne sont pas intervenues. Or la validité externe a «à voir avec l'aptitude à la généralisation: des résultats valides le seront-ils également pour une population élargie?» (RodRIK, 2008, p. 16, traduit par les auteurs).

L'abandon d'une expérimentation n'est pas chose facile même si l'article L. O. 1113-6 du Code des collectivités territoriales le prévoit comme issue possible. Cette réticence est exprimée par les évaluateurs du programme expérimental de 
l'académie de Créteil, sur le traitement de l'absentéisme scolaire. Ce programme, qui a défrayé la chronique, a reçu en avril 2010 un avis négatif à l'issue de sa phase pilote, aussitôt assorti de justifications (Behaghel, Gurgand, 2010, p. 16). Cet avis «ne signifie pas que le dispositif n'ait pas d'effets - seule l'évaluation d'impact permettrait de le montrer - mais que les conditions de l'évaluation ne sont pas remplies ». À cette occasion, les conditions d'acceptation sociale dont le rapport fait état ajoutent deux limites supplémentaires au cadre légitime de recours à l'expérimentation dans le domaine social : les questions évaluées doivent être consensuelles (alors que l'expérimentation randomisée est présentée canoniquement comme une façon de trancher des questions qui divisent l'opinion, faute de preuves), et les projets doivent être définis sans savoir qu'ils vont être financés dans le cadre d'une évaluation, ce qui nous semble en contradiction avec le principe même de l'appel à projets du Fonds d'expérimentation pour la jeunesse. Les évaluateurs ajoutent un autre type de condition (p. 2) : «du point de vue scientifique, force est de constater que les conditions d'une évaluation objective - où les acteurs ne modifient pas leur comportement en fonction de l'évaluation - n'ont pas été réunies au cours du pilote. Il n'y a pas de raison de penser que ces conditions puissent être réunies en 2010-2011». Savoir renoncer, tel est bien le défi auquel sont confrontés des expérimentateurs sociaux officiellement engagés dans la voie des réformes.

\section{Quelles contributions aux réformes?}

Un des traits remarquables des expérimentations sociales randomisées est leur vocation à être généralisées. Le Guide méthodologique pour l'évaluation des expérimentations sociales ${ }^{(20)}$, édité en 2009 à l'intention des porteurs de projets, en fait même une finalité première (p. 2) : «C'est une innovation de politique sociale initiée dans un premier temps à petite échelle, compte tenu des incertitudes existantes sur ses effets et mise en œuvre dans des conditions qui permettent d'en évaluer les résultats, dans l'optique d'une généralisation si ces résultats s'avèrent probants. » Dans son premier rapport d'évaluation, le conseil scientifique du fonds d'expérimentation estimait que le bilan de son action «se jugera[it] notamment à sa capacité à inspirer les décisions publiques» (MINISTĖRE DE L'ÉDUCATION Nationale, de La Jeunesse et de la Vie associative, 2011, p. 6).

(20) Le Guideméthodologiquepourl'évaluation des expérimentations sociales àl'intention des porteurs de projets est disponible en ligne à l'adresse : http://www.experimentation.jeunes.gouv. $\mathrm{fr} / \mathrm{IMG} / \mathrm{pdf} /$ guide-pour-l-evaluation-des-experimentations.pdf; consulté le 2 septembre 2013.
Si on prend au sérieux les promesses des expérimentateurs sociaux, on ne devrait pas tarder à voir naître des réformes entièrement construites à partir des résultats obtenus. C'est ce critère d'opérationnalité qui a dominé pendant cinq ans les investissements financiers dans ce type d'expérimentation. Il semble qu'aujourd'hui une prise de conscience ait lieu sur le réalisme de ces objectifs, et qu'on en revienne à une approche plus classique de l'évaluation.

\section{Des expérimentations aléatoires au service de la décision}

Très vite après leur promotion par la commission Hirsch, les expérimentations aléatoires ont trouvé leur place au sein du budget de l'État, sous la forme de deux fonds dédiés, le Fonds d'innovation et d'expérimentation sociale (FIES), créé en 2006, et le Fonds d'appui aux expérimentations en faveur des jeunes, plus connu sous l'appellation Fonds d'expérimentation pour la jeunesse (FEJ), créé en 2008.

Depuis la loi de finances pour 2006, le FIES accompagne le développement d'expérimentations en anticipation et en appui des politiques publiques en faveur des solidarités et de la cohésion sociale. Les expérimentations figurent au sein de la mission «Solidarité, insertion et égalité des chances», dans le programme 304 «Lutte contre la pauvreté: revenu de solidarité active et expérimentations sociales», au titre d'une action $\mathrm{n}^{\circ} 2$ dénommée «Expérimentations sociales et autres expériences en matière sociale et d'économie sociale ${ }^{(21)}$. Cette action est présentée comme une concrétisation de «la résolution $\mathrm{n}^{\circ} 13 \mathrm{du}$ rapport "Familles, vulnérabilité, pauvreté" de 2005 visant à faire de "l'audace, l'innovation et l'expérimentation" les mots-clés de l'action publique, à partir du constat que l'action auprès des familles vulnérables exigeait de faire du sur-mesure et d'innover» (p. 31). La caution scientifique est largement mise à contribution: «sélectionnées par un jury associant des personnalités qualifiées, les expérimentations soutenues par le fonds d'innovation et d'expérimentation sociale sont suscitées par appels à projets structurés autour des thèmes précités ${ }^{(22)}$ 》.

(21) Mission interministérielle projets annuels de performance (2012), Annexe au projet de loi de finances pour 2012. Solidarité, insertion et égalité des chances, «Programme 304. Lutte contre la pauvreté : revenu de solidarité active et expérimentations sociales», pp. 19-41. Disponible en ligne à l'adresse : http://www.performance-publique.budget.gouv.fr/ farandole/2012/pap/pdf/PAP2012 BG_Solidarite insertion egalite_des_chances.pdf; consulté le 2 septembre $2 \overline{0} 13$.

(22) Document de politique transversale. Projet de loi de finances pour 2011 (2011), Inclusion sociale, p. 17. Disponible en ligne à l'adresse : http://www.performance-publique.budget. gouv.fr/fileadmin/medias/documents/ressources/PLF2011/DPT/ DPT2011_inclusion_sociale.pdf; consulté le 2 septembre 2013. 
Quant au Fonds d'appui aux expérimentations en faveur des jeunes ${ }^{(23)}$, il inscrit les expérimentations dans le temps long de la recherche :

«Le fonds d'appui aux expérimentations en faveur des jeunes, institué par l'article 25 de la loi du $1^{\text {er }}$ décembre 2008 susvisée, a pour objet de financer des programmes expérimentaux visant à favoriser la réussite scolaire des élèves, à contribuer à l'égalité des chances et à améliorer l'insertion sociale et professionnelle durable des jeunes de moins de 25 ans. Le fonds peut à ce titre financer l'essaimage d'expérimentations dans de nouveaux territoires $[\ldots]^{(24)}$.»

Pour ses promoteurs, l'objectif du fonds n'est pas de financer des projets uniquement pour euxmêmes, mais également $d$ ' " apprendre, de manière à capitaliser des connaissances mobilisables pour concevoir les politiques publiques futures» (Ministère DE L'ÉduCATion NATIONALE, DE LA Jeunesse et de La Vie associative, 2011, p. 7). Les budgets et les actions suivent. Depuis sa création, le Fonds d'expérimentation pour la jeunesse (FEJ) a lancé 11 appels à projets, reçu plus de 1500 projets d'expérimentation, réuni plus de 30 jurys d'experts. Plus de 380 expérimentations (d'une durée moyenne de trois ans) ont été sélectionnées sur le territoire national (métropole et outre-mer) dans des thématiques extrêmement variées ${ }^{(25)}$.

Si l'action du FIES n'était pas assortie d'indicateurs dédiés, l'action du FEJ en comportait deux, déclinés pour le projet de loi de finances (PLF) 2012 dans le programme 163 «Jeunesse et vie associative», du projet annuel de performance (PAP) de la mission «Sport, jeunesse et vie associative». Le premier mesure la part des projets ayant démarré effectivement six mois après leur sélection, le second celle des "expérimentations contrôlées» dans l'ensemble des expérimentations. L'indicateur est assorti d'un commentaire qui réaffirme les principes et préceptes du guide méthodologique :

«[l'indicateur est] destiné à appuyer l'essor, au travers du FEJ, de la démarche d'expérimentation dite "contrôlée" qui repose sur l'observation d'un groupe test bénéficiant du dispositif et d'un groupe témoin ne bénéficiant pas du dispositif, ces groupes étant construits en y affectant les personnes par tirage au sort. Cette démarche d'évaluation quantitative, pratiquée notamment dans les pays anglo-saxons depuis plusieurs décennies reste en effet largement à développer en France, tout particulièrement dans le domaine des politiques publiques. S'appuyant sur des échantillons représentatifs (de quelques centaines à quelques milliers d'individus, voire davantage), elle

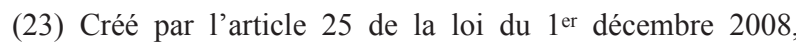
modifié par la loi n² 2010-1658 du 29 décembre 2010, art. 21.

(24) Décret $n^{\circ}$ 2011-1603 du 21 novembre 2011.

(25) Projets consultables à l'adresse http://www.experimentation.jeunes.gouv.fr/. assure une portée démonstrative forte au dispositif ainsi évalué» (p. 107).

L'insistance portée à la méthode atteint ici son paroxysme, jusqu'à laisser poindre le spectre d'une science officielle au service de l'action politique. Sans doute était-ce aller trop loin. Un changement de ton sensible se fait sentir dans les PAP associés au projet de loi de finances 2013, en direction d'une approche plus libre de l'intervention scientifique.

\section{Des expérimentations au service de la réflexion}

L'évolution de la présentation est particulièrement sensible pour les expérimentations inscrites dans le programme 304, «Lutte contre la pauvreté : revenu de solidarité active et expérimentations sociales». À compter de 2013, l'action 12 «Économie sociale et solidaire» de ce programme est dédiée exclusivement aux actions relatives au soutien et au développement de l'économie sociale et solidaire. Les actions portant sur les autres expérimentations en matière sociale sont inscrites sur une action 13 "Autres expérimentations», qui a changé considérablement de volume. Les actions du programme 304 se vident ainsi des crédits d'expérimentation : les 5981487 euros de crédits de l'ancienne action "Expérimentation sociale et autres expériences en matière sociale et d'économie sociale» pour 2012 abondent désormais pour 5000000 d'euros l'action 12 «Économie sociale et solidaire». Et les 981947 euros restants ne concernent plus les expérimentations que de manière lointaine :

«La dotation 2013 de $981487 €$ en $\mathrm{AE}=\mathrm{CP}^{(26)}$ permettra d'accompagner le développement d'expérimentations en anticipation et en appui des politiques publiques en faveur des solidarités et de la cohésion sociale.

Les crédits de l'action 13 permettent de soutenir le fonctionnement de l'Agence nouvelle des solidarités actives (ANSA), dans le cadre d'une convention pluriannuelle en cours, dans son objectif de contribuer au développement d'expérimentations, à la mutualisation de bonnes pratiques entre les territoires (notamment en matière d'accès aux droits des bénéficiaires de minima sociaux) et à l'expérimentation et l'évaluation de projets innovants dans le champ de la lutte contre la pauvreté, ciblés sur la prévention de la rupture du lien social.

Ils doivent permettre également le développement de démarches d'ingénierie sociale, dans le cadre d'un programme expérimental visant à tester la pertinence, l'efficacité, la cohérence et l'efficience d'une

(26) Les autorisations d'engagement (AE) constituent la limite supérieure des dépenses pouvant être engagées, et les crédits de paiement $(\mathrm{CP})$ constituent la limite supérieure des dépenses pouvant être ordonnancées ou payées pendant l'année pour la couverture des engagements contractés dans le cadre des autorisations d'engagement. 
politique publique de soutien à l'innovation sociale sur une échelle limitée (4 régions). Le programme aura pour objectif de renforcer la capacité de repérage et d'appui par les réseaux déconcentrés de la cohésion sociale des initiatives visant à renforcer le lien social dans les territoires, et à créer une dynamique autour de l'innovation sociale ${ }^{(27)}$.»

On ne saurait dire plus clairement qu'il s'agit désormais de soutenir des projets locaux, qui ont plus à voir avec l'expérimentation administrative qu'avec l'expérimentation à vocation scientifique.

Dans le PAP de la mission Sport, jeunesse et vie associative pour 2013, le ton change également dans la présentation des objectifs de l'expérimentation (28). Certes, l'objectif demeure «de soutenir et d'évaluer, selon des modalités précises, des dispositifs innovants concourant à 1'autonomie des jeunes, dans l'optique de la mise en place de nouvelles politiques publiques en direction des jeunes » (p. 89). Mais la méthode n'est plus évoquée qu'en passant, pour rappeler que "l'évaluation externe et scientifique de ces projets, si possible contrôlée, fait partie intégrante des conditions de sélection des projets financés » (p. 89). L'accent est mis sur la diffusion des travaux, comme une manière de renvoyer les expérimentateurs à une fonction d'information. Un nouvel indicateur 4.1 est élaboré, dont la finalité est de mesurer la diffusion des résultats des expérimentations soutenues : «afin d'apporter des éléments utiles de réflexion aux décideurs publics dans le cadre de l'élaboration des politiques de jeunesse, les résultats des expérimentations soutenues dans le cadre du FEJ doivent être disponibles et accessibles. Leur mise en ligne sur le site gouvernemental www.jeunes.gouv.fr constitue donc un enjeu important» (p. 89). L'indicateur comporte deux mesures : "Part des expérimentations ayant fait l'objet d'un rapport d'évaluation traité et publié / Nombre total d'expérimentations financées; Part des rapports finaux d'évaluation traités et publiés dans l'année / Nombre total de rapports attendus dans l'année » (p. 89). Dans la nouvelle terminologie, il s'agit désormais d'apporter des "éléments de réflexion», et non plus d'assurer une portée démonstrative forte. Dans ce

(27) Mission interministérielle projets annuels de performance (2013), Annexe au projet de loi de finances pour 2013. Solidarité, insertion et égalité des chances, p. 35. Disponible en ligne à l'adresse : http://www.performance-publique.budget. gouv.fr/farandole/2013/pap/pdf/PAP2013_BG_Solidarite insertion egalite des chances.pdf; consulté le $\overline{2}$ septembre 2013.

(28) Mission interministérielle projets annuels de performance (2012), Annexe au projet de loi de finances pour 2012. Sport, jeunesse et vie associative, "Programme 163. Jeunesse et vie associative », pp. 81-115. Disponible en ligne à l'adresse : http:// www.performance-publique.budget.gouv.fr/farandole/2013/ pap/pdf/PAP2013_BG_Sport_jeunesse_vie_associative.pdf; consulté le 2 septembre 2013. cadre, l'expérimentation aléatoire simple devient un simple réservoir d'idées, et n'est plus orientée vers des projets de réforme précis.

Enfin, de nouvelles expérimentations se profilent, qui relèvent d'une conception plus administrative de la mise à l'essai des normes. Au sein de la mission Solidarité, insertion, et égalité des chances, figure le programme 137 «Égalité entre les femmes et les hommes» dans lequel a été introduite pour 2013 l'action $\mathrm{n}^{\circ} 14 \mathrm{~d}^{\prime}$ '«Actions de soutien, d'expérimentation en faveur de l'égalité entre les femmes et les hommes» (p. 126) ${ }^{(29)}$. Dotée d'un budget de 6,3 millions d'euros, cette action comporte la création au 1 er janvier 2013 d'un fonds budgétaire "d'expérimentation en faveur des droits des femmes et de l'égalité entre les femmes et les hommes». Son objectif est de «mettre en œuvre des programmes de soutien et d'expérimentations et fonder les bases de nouvelles pratiques au service de l'égalité professionnelle et de la protection effective des femmes face aux violences» (p. 111 et 126).

Ces expérimentations sont définies par leur objet, sans indication de méthode ${ }^{(30)}$. Il n'est plus question de randomisation, mais de soutien à des dispositifs à l'essai, dans la grande tradition administrative.

Ce changement pourrait bien augurer une redistribution des responsabilités dans leurs champs respectifs : au décideur de décider, au scientifique de conduire des expérimentations, qu'il devra valider dans les règles de l'art. Il n'en demeurera pas moins que les chercheurs engagés dans l'expérimentation des réformes sont soumis au risque d'instrumentalisation de leurs résultats, comme ces chercheurs à qui a été commandée une expérimentation sur les curriculum vitce (CV) anonymes en

(29) Mission interministérielle projets annuels de performance (2012), Annexe au projet de loi de finances pour 2012. Solidarité, insertion et égalité des chances, «Programme 137. Égalité entre les femmes et les hommes», pp. 121-140. Disponible en ligne à l'adresse : http://www.performance-publique.budget.gouv.fr/ farandole/2012/pap/pdf/PAP2012_BG_Solidarite_insertion egalite_des_chances.pdf; consulté le 2 septembre 2013 .

(30) Elles porteront : «sur le développement des accords dans les entreprises et l'amélioration de leur qualité; sur l'orientation et la mixité pour développer la part des filles dans les filières scientifiques et techniques et leur part dans les métiers correspondants, mais aussi pour valoriser auprès des garçons des métiers à prédominance féminine; sur la formation des bénéficiaires du complément de libre choix d'activité pendant leur congé parental afin de réduire l'effet d'éloignement de l'emploi» (p. 110).

En outre, «d'autres expérimentations pourront être mises en place dans le champ de l'égalité professionnelle ou dans le champ de la lutte contre les violences, s'agissant notamment de l'extension de dispositifs de protection de type téléphone grande alerte.

Un programme d'expérimentations sera défini, s'appuyant sur des logiques de partenariat et d'accompagnement (avec les partenaires sociaux, associatifs, collectivités). Les programmes expérimentaux seront sélectionnés le plus souvent selon des procédures d'appel à projets» (p. 117). 
vue de la préparation d'un décret( ${ }^{31)}$ (BEHAGHEL et al., 2011). En excipant des résultats de l'étude, le commissaire à la diversité et à l'égalité des chances, Yazid Sabeg, renonçait à prendre le décret qui aurait rendu obligatoire l'anonymat des candidatures dans les entreprises concernées. Pourtant, les auteurs n'avaient nullement conclu «sur les effets complets d'une généralisation du CV anonyme», et précisaient que l'étude ne permettait pas de «tester l'existence de discriminations à l'embauche», comme ils l'avaient indiqué quelques mois auparavant, dans une longue lettre à un quotidien (32). On voit bien la difficulté des chercheurs à contrôler les interprétations données à leurs résultats par les commanditaires. Mais ce décalage n'a rien de surprenant : il atteste au contraire de l'autonomie de la justification politique par rapport à la justification scientifique, et doit conduire les chercheurs à chercher ailleurs que dans la décision politique la validation de leurs travaux.

\section{Trouver une place pour l'expérimentation sociale aléatoire}

La critique majeure qu'on peut formuler à l'encontre des expérimentations sociales «à la française», c'est d'avoir brouillé la frontière entre recherche et décision politique (Gomel, Serverin, 2009). Pour la rétablir, deux dimensions doivent être prises en compte: le souci éthique dans la conduite de la recherche, le nécessaire contrôle des expérimentations par les communautés scientifiques, au nom de l'indépendance de la recherche.

\section{La nécessaire prise en compte de l'éthique}

Quel type de contrôle éthique peut être exercé sur la recherche sociale expérimentale? En dehors des engagements éthiques pris par le chercheur lui-même au regard de l'instance (publique ou privée) qui l'emploie, on pense aux dispositifs d'autorisation et de contrôle qui encadrent la recherche sur l'homme, et notamment la recherche biomédicale. Ces règles sont-elles applicables aux expérimentations sociales? La réponse n'est pas immédiate. $\mathrm{Si}$, dans les codes, des règles existent bien sur l'éthique de la recherche expérimentale, elles sont centrées sur le vivant (corps humain et santé), et non sur le comportement humain. A priori, la définition donnée de la recherche

(31) L'article L. 1221-7 du Code du travail (modifié par une loi du 22 mars 2012), prévoit que «dans les entreprises d'au moins cinquante salariés, les informations mentionnées à l'article L. 1221-6 et communiquées par écrit par le candidat à un emploi ne peuvent être examinées que dans des conditions préservant son anonymat», et ajoute que «les modalités d'application du présent article sont déterminées par décret en Conseil d'État».

(32) Behaghel L., Crépon B., Le Barbanchon T. (2011), «CV anonyme : ce que dit l'évaluation», Libération, 27 avril 2011. biomédicale par l'article L. 1121-1 du Code de la santé publique (dans sa version issue de 1'ordonnance du 23 février 2010), ne semble pas adaptée à ce type de recherche : "Les recherches organisées et pratiquées sur l'être humain en vue du développement des connaissances biologiques ou médicales sont autorisées dans les conditions prévues au présent livre et sont désignées ci-après par les termes "recherche biomédicale" ". Pourtant, au détour de l'article L. 1121-3, alinéa 4 du même code, on trouve bien une référence (indirecte) à ce type d'expérience : «Dans les sciences du comportement humain, une personne qualifiée, conjointement avec l'investigateur, peut exercer la direction de la recherche ${ }^{(33)}$. »

Cette simple mention a trouvé un prolongement normatif avec la loi $n^{\circ} 2012-300$ du 5 mars 2012 relative aux recherches impliquant la personne humaine, issue de la proposition de loi soumise en 2009 par le député Jardé. Son auteur voulait donner un statut aux recherches non interventionnelles (comme le suivi de cohortes), pour leur permettre de passer par des comités d'éthique. La loi a introduit dans l'article L. 1121-1 du Code de la santé publique une distinction entre les recherches interventionnelles (qui supposent une intervention sur l'homme) et les recherches non interventionnelles, «dans lesquelles tous les actes sont pratiqués et les produits utilisés de manière habituelle, sans procédure supplémentaire ou inhabituelle de diagnostic, de traitement ou de surveillance ${ }^{(34)} \gg$.

Ce texte a fait basculer les recherches expérimentales sous le contrôle des comités de protection des personnes. Aucune expérimentation sur le comportement des personnes, qui revendique le qualificatif de «scientifique», ne devrait plus échapper à une présentation devant des instances éthiques. Ne pourraient en être exemptées que les expérimentations de type administratif, portant sur l'organisation des services, dont l'évaluation ne se veut pas scientifique, mais technique et administrative, et s'apparente aux études d'impact (Gomel, SERVERIN, 2011).

La loi vient ainsi au soutien de l'éthique, dans le sens indiqué depuis plusieurs années par le comité d'éthique du CNRS (Comets). Dans un premier avis de 2007, le comité recommandait d'être particulièrement prudent à l'égard des expérimentations sur les comportements qui peuvent faire courir un

(33) Cette précision, introduite par la loi $\mathrm{n}^{\circ} 94-630 \mathrm{du}$ 25 juillet 1994, a été maintenue par la loi du 7 juillet 2011 dans l'alinéa 4 du même article.

(34) L'article L. 1121-3 ajoute un septième alinéa qui précise que «les recherches non interventionnelles peuvent être effectuées sous la direction et la surveillance d'une personne qualifiée. Le comité de protection des personnes s'assure de l'adéquation entre la qualification du ou des investigateurs et les caractéristiques de la recherche.» 
risque psychique lorsqu'elles s'appliquent à des sujets vulnérables, comme «les sujets en difficulté sociale, immigrants, prisonniers, toxicomanes $\gg\left({ }^{(35)}\right.$. Un second avis de 2010, ciblé sur l'éthique de la recherche dans l'expérimentation sociale, recommandait également la soumission des projets à des principes éthiques, ainsi que leur publication dans des revues spécialisées dans les sciences expérimentales ${ }^{(36)}$.

\section{L'exigence d'un contrôle des résultats par les pairs}

Une recherche indépendante n'est pas synonyme de recherche sans contrôle. L'évaluation a toujours été inscrite au cœur des dispositifs institutionnels de la recherche publique, par deux procédés complémentaires : le contrôle de la dépense, et le contrôle de la pertinence. Le premier relève normalement de l'autorité publique qui alloue les fonds aux organismes de recherche; le second est confié à ce qu'on appelle communément les "communautés scientifiques». Ces principes d'évaluation se retrouvent dans un chapitre spécifique du Code de la recherche, «Évaluation et contrôle de la recherche et du développement technologique». L'article L. 114-1 indique notamment que «les activités de recherche financées en tout ou partie sur fonds publics, réalisées par des opérateurs publics ou privés, sont évaluées sur la base de critères objectifs adaptés à chacune d'elles et s'inspirant des meilleures pratiques internationales. Parmi ces critères, les contributions au développement de la culture scientifique [...] sont prises en compte». Ces évaluations s'effectuent en toute transparence, dans les conditions prévues par l'article L. 114-1-1: «Les procédures et résultats de l'évaluation d'une activité de recherche financée en tout ou partie sur fonds publics prévue à l'article L. 114-1 sont rendus publics dans des conditions assurant le respect des secrets protégés par la loi et des clauses de confidentialité figurant dans un contrat avec un tiers [...].»

Si les expérimentations sociales relèvent bien du domaine de la recherche scientifique, comme le revendiquent leurs promoteurs, et si on convient qu'elles sont financées sur fonds publics, elles devraient suivre les deux chemins de l'évaluation scientifique: l'évaluation par les pairs, par des publications dans des revues scientifiques comportant un comité de lecture; l'évaluation budgétaire des crédits qu'elles consomment, dont la proportion devrait être mesurée par rapport aux autres financements de la recherche publique. Le premier type d'évaluation suppose de définir clairement le champ disciplinaire couvert par les études (psychologie expérimentale, psychologie sociale, économie, statistique?) pour prendre la mesure des apports de connaissance. Quant à l'évaluation budgétaire, il semble, comme on l'a noté plus haut, que les finalités de la dépense se déplacent de la pure ingénierie sociale vers la connaissance. C'est là une évolution bienvenue. La dépense consacrée à l'expérimentation aléatoire pourrait alors revenir à la place qui lui revient naturellement dans le budget de l'État, c'est-à-dire dans la mission «Recherche et enseignement supérieur»».

(35) Comets, Réflexions sur éthique et sciences du comportement humain, avis du 23 février 2007, p. 23 et suivantes : «Un risque pour l'intégrité psychique existe chaque fois qu'un individu est soumis à des conditions pouvant altérer durablement son équilibre affectif [...]. On peut citer parmi ces conditions à risque [...] l'exposition à des stimuli émotionnels ou aversifs, l'utilisation de leurres ou de distorsions de la réalité (réalité virtuelle), la mise en échec systématique, la mise en compétition ou en conflit avec d'autres personnes. » Disponible en ligne à l'adresse : http://www.cnrs.fr/comets/IMG/pdf/14-comportement 070226-2.pdf; consulté le 2 septembre 2013.

(36) Comets, Ethique de la recherche dans l'expérimentation sociale, avis du 19 janvier 2010. Disponible en ligne à l'adresse : http://www.cnrs.fr/comets/IMG/pdf/07-experimentation-sociale-20100119-2.pdf; consulté le 2 septembre 2013. 


\section{Bibliographie}

Alberola E., Angotti M., Brézault M., Credoc (2008), «Enquête qualitative auprès des bénéficiaires du rSa, anciennement au RMI et à l'API, et de ceux qui n'ont pas pu y recourir. Synthèse des résultats intermédiaires », in Comité d'évaluation des expérimentations, Rapport d'étape sur l'évaluation des expérimentations rSa, Annexe 4. Disponible en ligne à l'adresse: http:// www.ladocumentationfrancaise.fr/var/storage/rapportspublics/084000607/0000.pdf; consulté le 2 septembre 2013.

Allègre G. (2008), «L'expérimentation sociale des incitations financières à l'emploi : questions méthodologiques et leçons des expériences nordaméricaines», Document de travail de l'OFCE, $\mathrm{n}^{\circ} 2008-22$.

ANPE, DARES, UnEDIC (2008), L'accompagnement renforcé des demandeurs d'emploi: l'évaluation des expérimentations, $\mathrm{n}^{\circ} 1$. Disponible en ligne à l'adresse : http://travail-emploi.gouv.fr/IMG/pdf/Evaluation OPPCVE_n1.pdf; consulté le 2 septembre 2013.

Avvisati F., Gurgand M., Guyon N., Maurin E. (2011), Quels effets attendre d'une politique d'implication des parents d'élèves dans les collèges? Évaluation de l'impact de la Mallette des parents, Rapport d'évaluation finale remis par l'École d'économie de Paris au Fonds d'expérimentation pour la Jeunesse dans le cadre de l'appel à projets lancé en 2007 par le ministère chargé de la Jeunesse, Paris, Ministère de l'Éducation nationale, de la Jeunesse et de la Vie associative. Disponible en ligne à l'adresse : http://www.experimentation.jeunes.gouv.fr/ IMG/pdf/APDIIESES_11_Rapport_final_evaluation Mallettes.pdf; consulté le 2 septembre 2013.

Behaghel L., Crépon B., Le Barbanchon T. (2011), Évaluation de l'impact du CV anonyme. Rapport final, mars. Disponible en ligne à l'adresse: http://www. crest.fr/ckfinder/userfiles/files/Pageperso/rapport_eval_ CVA_20110320.pdf; consulté le 2 septembre $201 \overline{3}$.

Behaghel L., Gurgand M. (2010), Programme expérimental "Bourses aux projets de classe» : bilan de la phase pilote du point de vue de l'évaluateur, Paris, École d'économie de Paris. Disponible en ligne à l'adresse : http://www.parisschoolofeconomics.eu/IMG/ pdf/Pilote-BoursesProjets-PSE-juin2010.pdf; consulté le 2 septembre 2013.

Behaghel L., Crépon B., Gurgand M. (2009), Évaluation d'impact de l'accompagnement des demandeurs d'emploi par les opérateurs privés de placement et le programme Cap vers l'entreprise. Rapport final, septembre. Disponible en ligne à l'adresse : http://travail-emploi. gouv.fr/IMG/pdf/Rapport_Final-_CREST-ENSEE.pdf; consulté le 2 septembre 2013.

Biais B., Rullière J.-L. (2003), «Approches expérimentales en économie et en finance», Lettre du département Sciences de l'Homme et de la société, CNRS, n 66 , pp. 48-50.
Cahuc P., Zylberberg A. (2009), Les réformes ratées du président Sarkozy, Paris, Flammarion.

Comité D’évaluation des eXPÉRIMEnTATIOns (2008), Rapport d'étape sur l'évaluation des expérimentations $r S a$. Synthèse, septembre. Disponible en ligne à l'adresse : http://lesrapports.ladocumentationfrancaise.fr/ BRP/084000607/0000.pdf; consulté le 2 septembre 2013.

Comité D'Évaluation des EXPÉRIMENTATIONS (2009), Rapport final sur l'évaluation des expérimentations $r S a$, mai. Disponible en ligne à l'adresse : http://www. ladocumentationfrancaise.fr/var/storage/rapportspublics/094000222/0000.pdf; consulté le 2 septembre 2013.

Commission Famille, vulnérabilité, pauvreté (2005), Au possible nous sommes tenus. La nouvelle équation sociale: 15 résolutions pour combattre la pauvreté des enfants, Paris, Ministère des Solidarités, de la Santé et de la Famille. Disponible en ligne à l'adresse : http:// www.ladocumentationfrancaise.fr/var/storage/rapportspublics/054000264/0000.pdf; consulté le 2 septembre 2013.

FISHER R. A. (1935), The design of experiments, Edinburgh, London, Oliver and Boyde.

Gomel B., Serverin E. (2009), «Expérimenter pour décider? Le RSA en débat», Document de travail, $\mathrm{n}^{\circ} 119$, Noisy-le-Grand, Centre d'études de l'emploi.

Gomel B., Serverin E. (2011), «Évaluerl'expérimentation sociale», Document de travail, $\mathrm{n}^{\circ} 143$, Noisy-le-Grand, Centre d'études de l'emploi.

JANIN P. (2005), «L'expérimentation juridique dans l'acte II de la décentralisation. Observations sur une réforme», La semaine juridique - Administrations et collectivités territoriales, $\mathrm{n}^{\circ}$ 41, p. 1334.

Lévy-Lambert H., Guillaume H. (1971), La rationalisation des choix budgétaires, Paris, Presses universitaires de France, coll. «SUP l'économiste».

Loncle P., Muniglia V., Rivard T. (2008), «La mise en œuvre de l'expérimentation du RSA à partir des enquêtes qualitatives réalisées dans cinq départements », in COMITÉ D'ÉVALUATION DES EXPÉRIMENTATIONS (2009), Rapport final sur l'évaluation des expérimentations $r \mathrm{Sa}$, Annexe 3.

Ministère de L'ÉducAtion NATIOnAle, de la JeUnesse ET DE LA VIE ASSOCIATIVE (2011), Rapport du conseil scientifique du Fonds d'expérimentation pour la jeunesse pour la période mai 2009-décembre 2010, Paris, Ministère de l'Éducation nationale, de la Jeunesse et de la Vie associative. Disponible en ligne à l'adresse : http:// www.experimentation.jeunes.gouv.fr/IMG/pdf/rapportcs-fej-2010.pdf; consulté le 2 septembre 2013.

PARIENTÉ W. (2008), «Analyse d'impact: l'apport des évaluations aléatoires », STATECO, $\mathrm{n}^{\circ} 103$.

RoDrik D. (2008), « The new development economics: we shall experiment, but how shall we learn? », John F. 
Kennedy School of Government, Harvard University, revised paper for the Brookings Development Conference.

Salvat X., Boccon-Gibod D. (2013), Rapport à Madame la garde des sceaux, ministre de la justice sur l'expérimentation des citoyens assesseurs dans les ressorts des cours d'appel de Dijon et Toulouse, février, Paris, Ministère de la Justice. Disponible en ligne à l'adresse : http://www.ladocumentationfrancaise.fr/var/ storage/rapports-publics/134000144/0000.pdf; consulté le 2 septembre 2013.
SERVERIN E. (2012), «Les causes et les effets du non-recours au RSA activité», Revue de droit sanitaire et social, $\mathrm{n}^{\circ} 4$, pp. 637-645.

Stahl J.-H. (2010), «L'expérimentation en droit français : une curiosité en mal d'acclimatation», Revue juridique de l'économie publique, $\mathrm{n}^{\circ} 681$.

Viveret P. (1990), L'évaluation des politiques et des actions publiques. Rapport au Premier ministre, Paris, La Documentation française. 